\title{
Session 7: Infectious diseases I
}

\author{
Tuesday 30th October 2007. Moderator: Alois Lang
}

[14.00-14.45]

\author{
Human monoclonal antibodies against HCV: A \\ clinical perspective \\ Roberto Burioni \\ Università Vita Salute San Raffaele, Milan, Italy
}

\begin{abstract}
The humoral and cellular response of the host against a virus is a natural selective constraint operating during virus replication in vivo. While acutely infecting viruses have the chance of escaping the host in a very short time, viruses persistently infecting the host are forced to cope with the adaptive host immune response, that the virus itself must modulate, disrupt, or evade with genetic variation. The ability of the virus to escape from cytotoxic $\mathrm{T}$ cells and neutralizing antibodies without marked reduction in fitness can be crucial for persistence and disease progression. Antibody response is particularly intriguing, as recent technological advances made possible the generation of a large number of human or humanized monoclonal antibodies (Mabs), that can be safely administered to patients and that can constitute a novel class of drugs for antiviral therapy. Virus that are persistently infecting the host are usually endowed with regions that can elicit neutralizing antibodies, but these regions evolved in such a way that can be easily mutated without affecting viral fitness. Availability of large panels of human Mabs clarified the nature of the human humoral response against persistent viruses such as $\mathrm{HCV}$, indicating how neutralizing antibodies that are recognizing conserved regions are present in the host repertoire, even if in a minor proportion. Once cloned and produced, these molecules can constitute a novel class of antiviral drugs capable of targeting multiple viral structures.
\end{abstract}

\section{[14.45-15.15]}

Immunoglobulin gene usage for neutralizing human monoclonal antibodies directed against the V3 region of $\mathrm{HIV}-1$
Miroslaw K.Gorny ${ }^{\mathrm{a}}$, Xiao-Hong Wang ${ }^{\mathrm{b}}$, Constance Williams $^{\mathrm{a}}$, Barbara Volsky ${ }^{\mathrm{a}}$, Kathy Revesz ${ }^{\mathrm{b}}$, Tetsuya Kimura $^{a}$ and Susan Zolla-Pazner ${ }^{a, b}$

${ }^{a}$ New York University School of Medicine, New York, $N Y, U S A$

${ }^{\mathrm{b}}$ Veterans Affairs New York Harbor Healthcare System, New York, NY, USA

There is great interest in studying the anti-V3 antibodies (Abs) due to their capacity to both neutralize primary isolates and their potential to play a protective role against HIV infection. A number of human anti-V3 monoclonal Abs (mAbs) were generated in our laboratory from HIV-1 infected individuals in order to study the mechanism of neutralization and to characterize the $\mathrm{V} 3$ region on the virus envelope. The human anti-V3 mAbs exhibit a broad range of activity; they can be type-specific, reactive with a limited number of viruses, or the mAbs can be broadly cross-reactive and bind to and/or neutralize viruses representing many HIV-1 subtypes. We postulated that this broad range of activity may depend on their genetic background which can be analyzed by examining their immunoglobulin (Ig) gene usage.

Our studies have shown that there is a biased usage of the VH gene families by human anti-HIV-1 mAbs. The anti-V3 mAbs, which were primarily sequenced in our laboratory, preferentially used the VH5-51 gene segment. However, based on our analysis of the published data, the anti-CD4-binding domain, the anti-CD4 induced antigen and the anti-gp41 mAbs preferentially used the VH1 gene family. The VH3 gene family was used at a significantly lower frequency by all of the analyzed anti-HIV-1 mAbs while the VH2, VH4 and VH6 gene families were used within a normal range of the repertoire.

We have tested the hypothesis that the neutralizing activity of anti-V3 mAbs against primary isolates may be related to different $\mathrm{VH}$ gene usage for the mAbs. The neutralizing activity of eight VH5-51 V3 mAbs 
was compared with that of eight non-VH5-51 V3 mAbs in assays using viruses pseudotyped with the envelope proteins from either subtype B (psSF162 and ps6535) or subtype C (psMW965) primary isolates. The results were statistically analyzed using the inhibitory doses of $\mathrm{mAbs}$ for $50 \%$ neutralization $\left(\mathrm{IC}_{50}\right)$. The VH5-51 V3 mAbs displayed significantly more efficient neutralizing activity against the three pseudotyped viruses than did the non-VH5-51 V3 mAbs. These data support the concept of a relationship between Ig structure, as determined by Ig genes, and antibody function.

\section{[15.15-15.35]}

\section{Antibodies mediating protection by a $\beta$-glucan} conjugate vaccine and with direct fungicidal activity: The isotype makes the difference Antonella Torosantucci, Paola Chiani, Carla Bromuro and Antonio Cassone

Department of Infectious, Parasitic and Immunomediated Diseases ISS, Rome, Italy

A vaccine made up by an algal $\beta$-glucan (laminarin), conjugated with a protein component, protects against infections by different human opportunistic pathogenic fungi and induces antibodies capable of inhibiting fungal growth [1]. Although taking a premium on a common and highly -conserved molecular theme, this remains a sort of "cross-kingdom" vaccine because the immunizing antigen and the vaccination target belong to organisms from two different kingdoms, and this is certainly the first case in the field of human vaccines. Thus, it is possible to convey in a single immunological tool the potential to protect against multiple infections, in theory all those caused by $\beta$-glucan-expressing fungi.

To obtain a better insight into the mechanisms of antibody protection of this sort of "universal" antifungal vaccine, a number of murine anti- $\beta$-glucan mAbs were generated, sequenced and characterized. Two mAbs (2G8 and 1E12) with identical CDR sequences but belonging to $\operatorname{IgG} 2 \mathrm{~b}$ and $\operatorname{IgM}$, respectively, and both efficiently binding and opsonising Candida albicans as test model, markedly differed in their capacity to passively vaccinate against the fungus. Namely, the $\operatorname{IgG} 2 \mathrm{~b}$ was highly protective whereas theIgM was non-protective in a systemic infection model. Affinity binding studies and ELISA inhibition assays showed that the mAb 2G8 recognized at high affinity oligosaccharides with $\beta 1-3$ configuration whereas the $\mathrm{mAb} 1 \mathrm{E} 12$ recognized efficiently both $\beta 1-3$ and $\beta 1-6$ oligosaccharide con- figurations. Importantly, the IgG2b mAb efficiently inhibited the in vitro growth of the hyphal, virulent forms of C.albicans, a property not shared by the IgM mAb. Glucan-associated cell wall proteins (GAP) are the likely targets for growth inhibition of fungal cells, and a high molecular weight GAP is bound by the $\mathrm{mAb}$ $2 \mathrm{G} 8$ and is presently being identified by mass spectrometry. Overall, our data point to the critical function of antibody isotype in the mechanism of the direct, non immunoeffector-mediated, fungistatic activity of anti- $\beta$-glucan antibodies.

\section{References}

[1] Torosantucci et al., A novel glycoconjugate vaccine against fungal pathogens, J Exp Med 202 (2005), 597-606.

\section{[15.35-15.55]}

Use of a novel hetero-hybridoma method to clone potent, high affinity human antibodies specific for botulinum neurotoxins

Scott K. Dessain*, Sharad P. Adekar, R. Mark Jones, M. D. Elias, Fetweh H. Al-Saleem, Michael J. Root and Lance L. Simpson

Cardeza Foundation for Hematologic Research and the Kimmel Cancer Center of Thomas Jefferson University, 1015 Walnut St., Philadelphia, PA 19107, USA

*Presenting author: E-mail: scott.dessain@jefferson.edu

Hybridoma methods of human antibody cloning have many potential advantages, including technical simplicity, high-level antibody production, and expression of antibodies in their native configurations. However, they have been hampered by inefficiency and the limited accessibility of antigen-specific human B-cells. We have created a novel fusion partner cell line, a murine hybridoma cell line that ectopically expresses the human telomerase (hTERT) and murine interleukin6 (mIL-6) genes. Hybrids derived from this cell line and primary human B-cells are stable and express high levels of human antibodies. We have optimized methods for the use of this fusion partner cell line in order to clone human antibodies specific for botulinum neurotoxin (BoNT). BoNT is a category A select bioterror agent and the most potent biological toxin known. BoNT exists in 7 serotypes, each of which may require specific therapeutic countermeasures.

We obtained peripheral blood mononuclear cells from volunteers vaccinated with the pentavalent bo- 
tulinum toxoid vaccine (serotypes A-E). From cell fusion experiments, we have cloned human IgG antibodies specific for BoNT serotypes A, B and E. Two of the antibodies, 6A and 13A, have high affinity for BoNT/A, with solution $\mathrm{K}_{D}$ values estimated to be $6.9 \times 10^{-12} \mathrm{M}$ and $9.7 \times 10^{-11} \mathrm{M}$, respectively. In the standard mouse protection assay, the $6 \mathrm{~A}$ BoNT/A antibody is able to protect mice from a lethal dose of BoNT/A. These experiments demonstrate the utility of a novel human hybridoma method for cloning active, antigenspecific antibodies with extremely high affinities, using peripheral blood B-cells as starting material. 